\title{
Exodoncia en pacientes geriátricos con bifosfonatos
}

\section{Extraction in geriatric patients with phosphonates}

\author{
Martínez-Rodríguez $\mathrm{N}^{*}$, Rubio-Alonso LJ**, Leco-Berrocal I***, \\ Barona-Dorado C****, Martínez-González JM*****
}

\section{RESUMEN}

En el paciente geriátrico, resulta común la presencia de enfermedades sistémicas, lo que conlleva a un consumo de diferentes fármacos para el tratamiento de las mismas. Uno de los más frecuentes son los bifosfonatos. Por lo general, los bifosfonatos son fármacos bien tolerados si se administran correctamente, aunque en los últimos años también se han descrito diferentes efectos adversos asociados a su consumo, siendo la osteonecrosis maxilar uno de lo más importantes. Desde entonces son numerosos los casos publicados en los cuales se afirma que los procedimientos quirúrgicos menores como las exodoncias podrían ser uno de los factores desencadenantes. En el caso de los bifosfonatos intravenosos, dado que el riesgo es mucho mayor, se sugiere evitar en lo posible los tratamientos quirúrgicos orales como las extracciones mientras que en los tratamientos con bifosfonatos orales no se puede contraindicar dichos tratamientos de esa manera tan categórica Ante esta situación resulta evidente la necesidad de establecer unos protocolos definitivos encaminados a la prevención y tratamiento de esta posible complicación que pudiese acontecer.

Palabras clave: Exodoncia, bifosfonatos, maxilar, mandíbula.

\section{SUMMARY}

In geriatric patients is common the presence of systemic diseases, leading to a use of various drugs for the treatment thereof. One of the most common are the bisphosphonates. In general, bisphosphonates are well tolerated drugs if properly managed, although in recent years have also been described different adverse effects associated with its consumption, with the maxillary osteonecrosis one of the most important. Since then are numerous reported cases in which it is stated that minor surgical procedures such as extractions could be one of the triggers. In the case of intravenous bisphosphonates, since the risk is much higher, it is suggested to avoid possible oral surgical treatments like extractions while on treatment with oral bisphosphonates may not contraindicate such treatments that so categorically. In this situation the need to establish a definitive protocols aimed at the prevention and treatment of this possible complication that could happen is obvious. Key words: Extraction, biphosphonate, jaws.

Fecha de recepción: 2 de mayo 2015.

Aceptado para publicación: 6 de mayo 2015.

* Profesora contratada del Máster de Cirugía Bucal. Hospital Virgen de la Paloma. Madrid. Profesora de Clínica Integrada de Adultos y Patología Quirúrgica Bucal. Facultad de Ciencias de la Salud. URJC.

** Profesor contratado del Máster de Cirugía Bucal. Hospital Virgen de la Paloma. Madrid. Profesor Colaborador Honorífico de Cirugía Bucal. Facultad de Odontología. UCM.

*** Profesora contratado del Máster de Cirugía Bucal. Hospital Virgen de La Paloma. Madrid. Profesora Asociada de Cirugía Bucal. Facultad de Odontología. UCM.

**** Subdirectora del Máster Cirugía Bucal. Hospital Virgen de la Paloma. Madrid. Profesora Asociada de Cirugía Bucal. Facultad de Odontología. UCM.

***** Profesor Titular de Cirugía Maxilofacial. Facultad de Odontología. UCM.

Martínez-Rodríguez N, Rubio-Alonso LJ, Leco-Berrocal I, Barona-Dorado C, Martínez-González JM. Exodoncia en pacientes geriátricos con bifosfonatos. Av. Odontoestomatol 2015; 31 (3): 173-179. 


\section{INTRODUCCIÓN}

La extracción dentaria en el paciente geriátrico representa el tratamiento más frecuente en cirugía bucal, dada la habitual presencia de caries y enfermedad periodontal.

En estos pacientes resulta común la presencia de enfermedades sistémicas, lo que conlleva a un consumo de diferentes fármacos para el tratamiento de las mismas. Este hecho puede repercutir sobre la salud bucodental y debe ser tenido en cuenta a la hora de efectuar cualquier tratamiento quirúrgico.

Uno de los tratamientos más frecuentes son los bifosfonatos, fármacos análogos de los pirofosfatos endógenos que se fijan al hueso actuando como potentes inhibidores de la actividad osteoclástica. Su uso va encaminado a la prevención y tratamiento de enfermedades que cursen con reabsorción ósea, ya sea de tipo oncológico o no oncológico como la osteoporosis (1).

Por lo general, los bifosfonatos son fármacos bien tolerados si se administran correctamente, aunque en los últimos años también se han descrito diferentes efectos adversos asociados a su consumo. Dentro de estos cabe destacar los gastrointestinales como náuseas dispepsia, dolor abdominal, alteraciones renales o síntomas pseudogripales. Los efectos adversos a nivel oral asociados incluyen ulceraciones orales en la mucosa así como osteonecrosis de los maxilares (1-3).

La osteonecrosis de los maxilares por bifosfonatos fue descrita en el año 2003, en una serie de pacientes oncológicos o con trastornos metabólicos tratados por vía endovenosa. Desde entonces son numerosos los casos publicados en los cuales se afirma que los procedimientos quirúrgicos menores como las exodoncias podrían ser uno de los factores desencadenantes (4-7).

Ante el considerable aumento en el consumo de estos fármacos en pacientes mayores así como a su uso de forma prolongada, resulta evidente la necesidad de establecer unos protocolos encaminados a la prevención y tratamiento de las posibles complicaciones que pudiesen acontecer.

\section{MECANISMO DE ACCIÓN DE LOS BIFOSFONATOS}

Los bifosfonatos son compuestos que tienen una baja absorción intestinal, pero una alta y selectiva captación y retención a nivel óseo. Se estima que aproximadamente el $80 \%$ es eliminado por el riñón y el $20 \%$ captados por el hueso. Estos fármacos presentan en su composición química dos átomos de fósforo unidos a un átomo de carbono (P-C-P). Esta estructura les confiere una resistencia a la hidrólisis enzimática y les permite fijarse con gran avidez a la superficie de los cristales de hidroxiapatita con una rápida y eficiente unión del fármaco a la superficie mineral ósea (8-10).

Su efecto antirresortivo hace que actúen directamente sobre los mecanismos mediados por los osteoblastos que controlan la activación de los osteoclastos, interfiriendo directamente sobre estos en su proliferación, diferenciación, maduración, implantación ósea y finalmente en su apoptosis (11).

En función del tipo de bifosfonato, el mecanismo es diferente, mientras que los bifosfonatos simples, se acumulan en el interior de los osteoclastos como análogos no hidrolizables del adenosín trifosfato (ATP) induciendo la apoptosis, los aminobifosfonatos inhiben la vía del mavelonato; concretamente la enzima farnesil difosfato sintetasa en los osteoclastos. Además, éstos acaban produciendo la inhibición de los lípidos isoprenoides esenciales para la farnesilación y geranilación de pequeñas proteínas GTPasa señalizadoras. La pérdida de estas proteínas señalizadoras es decisiva para la disminución de la actividad reabsortiva y la apoptosis de los osteoclastos (12).

De este forma se producen unos cambios en la estructura ósea que permiten aumentar sus propiedades estructurales y por tanto provocando un efecto antifractura. Este efecto ha podido ser objetivado en varios estudios aleatorizados con largos periodos de seguimiento en pacientes osteoporóticos (13).

Los bifosfonatos también son capaces de inhibir la actividad de otros muchos factores que intervienen sobre la resorción ósea como son la paratohormona, las prostaglandinas, los derivados retinoides y 
diferentes citoquinas que incluyen las pertenecientes a la familia de las interleuquina, el Factor de Necrosis Tumoral (FNT), el Factor de Crecimiento Transformado (FCT) (14-15).

\section{CLASIFICACIÓN DE LOS BIFOSFONATOS}

Los bifosfonatos se diferencian por sustituir el oxígeno central del pirofosfato por un átomo de carbono. (Figura 1) Los distintos tipos se diferencian por los diversos componentes de las cadenas R1 y R2, lo que también influye en su potencia.

Los que no contienen una molécula de nitrógeno se conocen como bifosfonatos simples o de $1^{\mathrm{a}}$ generación y los que sí la tienen en su fórmula se les denomina aminobifosfonatos o de $2^{a}$ generación. Este último grupo poseen una actividad mucho más potente ya que se acumulan en la matriz ósea y en los osteoclastos en concentraciones máximas durante las primeras 24-48 horas, permaneciendo en el hueso mucho más tiempo (16).

Otro aspecto a tener en cuenta a la hora de valorar la potencia de acción de los bifosfonatos es el hecho de que éstos, por vía oral, se absorben relativamente mal, por lo que la biodisponibilidad de los bifosfonatos orales es menor al $2 \%$ que la de los administrados por vía parenteral.

\section{INDICACIONES DE LOS BIFOSFONATOS}

En la actualidad, la principal utilidad clínica del uso de los bifosfonatos se aplica en aquellas enfermedades en la que exista un aumento importante de la reabsorción ósea, tales como las hipercalcemias,

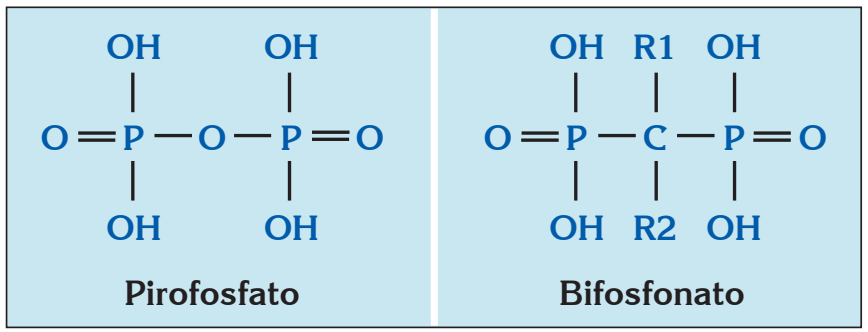

Fig. 1. Estructura química del pirofosfato y los bifosfonatos. especialmente las de origen tumoral y las metástasis óseas por cáncer, la enfermedad de Paget y la osteoporosis sobretodo en mujeres postmenopáusicas. De la misma manera se indican como agentes terapéuticos en casos de calcificación distrófica y en la osificación heterotópica, dados sus efectos en animales de experimentación sobre la prevención de calcificaciones aórticas, renales y dérmicas inducidas por alta dosis de vitamina $\mathrm{D}$ o de la calcificación periarticular vista en la artritis experimental $(2,3,5,11)$.

Adicionalmente se ha comprobado su utilidad sobre el síndrome de la distrofia simpática refleja, en la osteoartritis así como en enfermedades del metabolismo óseo hereditario que incluyen la osteogénesis imperfecta, la displasia fibrosa, la osteopatía hipertrófica, la mastocitosis sistémica, la enfermedad de Gaucher y la hiperostosis esternocostoclavicular (17).

\section{REPERCUSIONES DE LOS BIFOSFONATOS EN LA EXODONCIA}

En los pacientes que están en tratamiento con bifosfonatos, y debido a su acción antiosteoclástica, la capacidad de regeneración y reparación ósea del alveolo dental tras una extracción dental, puede verse alterada (Figuras 2 y 3). En algunos casos, tras extracciones y/o cirugías dentales, el proceso de cicatrización que normalmente debe producirse, no lo hace, y el hueso maxilar o mandibular queda expuesto (16).

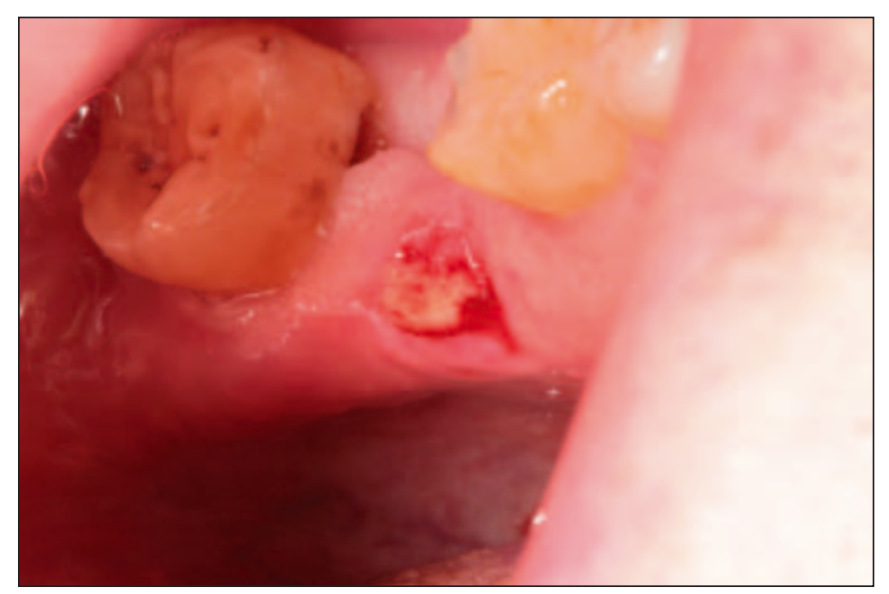

Fig. 2. Osteonecrosis lingual postextracción, estadio 1. Antecedentes de ingesta de alendronato. 


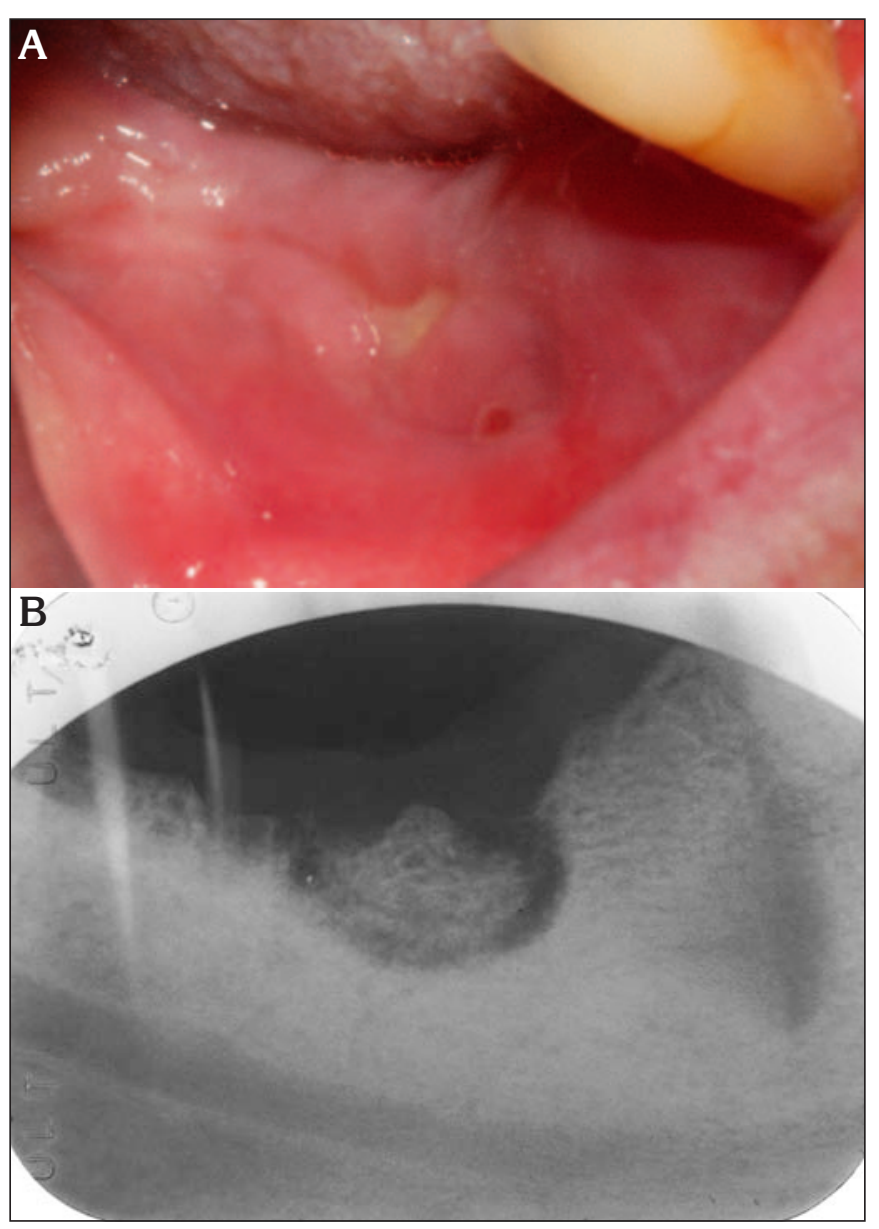

Fig. 3. Osteonecrosis con antecedentes de ingesta de risedronato. A) Exposición ósea con supuración (estadio 2). B) Imagen radiográfica del secuestro óseo.

Este fenómeno es conocido como la osteonecrosis inducida por bifosfonatos que fue definida en el año 2007 por la Asociación Americana de Cirujanos Orales y Maxilofaciales (AAOMS) como la presencia de exposición ósea en los maxilares durante más de ocho semanas en pacientes que hayan sido tratados con estos fármacos y que no hayan recibido radioterapia (18).

La mayoría de los estudios coinciden en que los únicos huesos afectados son los maxilares, siendo la mandíbula la más frecuente debido a su vascularización terminal. Además los maxilares están expuestos al medio externo a través del espacio periodontal y tienen un recambio mucho más elevado que el resto del esqueleto, en especial la cresta alveolar. Si a estos factores les añadimos la alta frecuencia de infecciones óseas de etiología dentaria, tratamientos odontológicos y la fina mucosa que recubre el hueso maxilar, explicarían porqué esta condición osteonecrótica se manifiesta especialmente en esta zona del organismo $(2,7)$.

Del mismo modo, esta posible complicación se ha observado sobretodo con el uso de bifosfonatos intravenosos, como es el caso del ácido zoledrónico donde la incidencia está comprendida entre el 1$15 \%$ de los pacientes que reciben dicha medicación. Por el contrario, se produce muy rara vez con los bifosfonatos orales, habiéndose descrito con estos últimos una posible incidencia de 1 caso de cada 10.000-100.000 pacientes que lo toman. Así son varios los estudios que han revelado su escasa repercusión en el caso de que estos fármacos se utilicen por vía oral, para tratamientos como la osteoporosis y otros procesos reumatológicos. Sin embargo otros autores afirman que entre un $20 \%$ y un $40 \%$ de los casos acontecen de forma espontánea (7,17-19).

Uno de los interrogantes que se ha planteado en la actualidad, es que no se puede predecir qué pacientes van a desarrollar osteonecrosis por bifosfonatos. En este sentido parece evidente, que para que ésta se produzca debería existir un factor desencadenante. Son varios los estudios que atribuyen a los procesos quirúrgicos bucales menores del tipo exodoncias, cirugías dentoalveolares o implantes como el principal factor desencadenante. Según varios estudios, entre un $52 \%$ y un $61 \%$ de los casos habían sido sometidos a una extracción previa (20). Es por esto por lo que algunos autores coinciden en suspender el tratamiento con bifosfonatos antes de realizar cualquier intervención de cirugía oral, pero no hay evidencia científica que nos confirme un menor riesgo de osteonecrosis, ya que la vida media de los bifosfonatos en el hueso es muy larga.

En el caso de los bifosfonatos intravenosos, dado que el riesgo es mucho mayor, se sugiere evitar en lo posible los tratamientos quirúrgicos orales, tales como extracciones o implantes, mientras que en los tratamientos con bifosfonatos orales no se puede contraindicar dichos tratamientos de esa manera tan categórica.

Uno de los objetivos principales como se ha señalado anteriormente sería el poder predecir qué pacientes son susceptibles de desarrollar una osteonecro- 
sis. Marx y cols. (21) y posteriormente Hutcheson y cols. (22) propusieron la determinación del CTX en suero (telómero C-terminal del colágeno 1), señalando que aquellos pacientes que han tomado bifosfonatos orales más de tres años y tienen cifras de CTX por debajo de $150 \mathrm{pg} / \mathrm{ml}$, serían los de mayor riesgo ante cirugías dentales. Sin embargo, se necesitan más estudios que ratifiquen estas afirmaciones ,tal y como sugieren Ruggiero y cols. (23).

\section{PROTOCOLO DE ACTUACIÓN ANTE PACIENTES EN TRATAMIENTO CON BIFOSFONATOS}

Existe un consenso por parte de la comunidad científica en la necesidad de informar a todo paciente en tratamiento con bifosfonatos de los posibles riesgos que de estos pudieran derivarse (23). Se puede agrupar a los pacientes en función de si van a comenzar con el tratamiento o por el contrario si están bajo el mismo en el momento de realizar la extracción.

\section{Pacientes antes de recibir tratamiento con bifosfonatos}

En aquellos casos de pacientes que vayan a ser sometidos a tratamiento con bifosfonatos resulta imprescindible que sean examinados clínica y radiológicamente de forma minuciosa ya que de este modo se ha observado una disminución significativa del riesgo de osteonecrosis. En estos casos aconsejan realizar tartrectomías así como instruir al paciente para que lleve una higiene oral rigurosa y realizar todos aquellos tratamientos conservadores pertinentes así como la extracción de los dientes no viables o con un pronóstico incierto e incluso la colocación de implantes si así se requiere $(23,24)$. Además se aconseja retrasar el inicio del tratamiento hasta que la zona de la extracción esté bien cicatrizada.

\section{Pacientes en tratamiento con bifosfonatos}

La suspensión del bifosfonato de tipo oral para la realización de una exodoncia ha generado mucha controversia. En la actualidad se considera que aque- llos pacientes que hayan estado en tratamiento con bifosfonatos con una duración menor a los cuatro años y que no tengan ningún factor de riesgo añadido se les podría realizar cualquier tipo de intervención quirúrgica.

Por otro lado, la Asociación Americana de Cirujanos Orales Maxilofaciales (AAOMS) sugería la suspensión del fármaco tres meses antes y dos meses después, en aquellos pacientes que hubiesen estado en tratamiento con estos fármacos desde más de tres años. Sin embargo, en 2014, la AAOMS, en su último documento de consenso sobre la osteonecrosis por bifosfonatos (23), recomendaron una suspensión del fármaco dos meses antes de la cirugía, siempre y cuando la condición sistémica lo permita, en aquellos pacientes en tratamiento de más de cuatro años así como aquellos que presentasen factores de riesgo concomitantes como la artritis reumatoide, previa o actual exposición a corticoides, los diabéticos así como las fumadores hasta que el alveolo estuviese cicatrizado correctamente (19).

Los pacientes que reciben bifosfonatos mensuales por vía intravenosa para el tratamiento de procesos oncológicos, son los que mayor riesgo representan de desarrollo de complicaciones. Es por esto por lo que cualquier tipo de procedimiento quirúrgico estaría contraindicado y se aconseja en casos de dientes no restaurables eliminar la corona del mismo y realizar la endodoncia de los conductos (23).

Si no existiese otra posibilidad que no fuese la exodoncia se aconseja realizarla lo más atraumáticamente posible, suturando el alveolo con el objetivo de favorecer la cicatrización. También sería conveniente prescribir una profilaxis antibiótica con amoxicilina/ ácido clavulánico (875/125 mg) o clindamicina (300 $\mathrm{mg}$ ) en el caso de alérgicos a penicilina desde dos días antes de la extracción y durante los diez días posteriores, así como enjuagues con clorhexidina al $0,12 \%$ dos veces al día (25).

\section{CONCLUSIONES}

El odontólogo y el cirujano bucal deben estar familiarizados con estos fármacos, de uso común en el paciente geriátrico, y prestar la atención suficiente 
para prevenir y detectar de forma temprana las posibles complicaciones que pueden acontecer como consecuencia de su consumo.

Son necesarios más estudios clínicos que permitan establecer un protocolo definitivo tanto de prevención como de tratamiento.

\section{BIBLIOGRAFÍA}

1. Mínguez-Serra MP, Salort-Llorca C, SilvestreDonat FJ. Oral Implant in patients receiving bisphosphonates: A review and update. Med Oral Patol Oral Cir Bucal 2008;1:13(12):E75560.

2. Bagán JV, Murillo J, Jiménez Y, Poveda E, Millán MA, Sanchís JM, et al. Jaw osteonecrosis associated with bisphosphonates: Multiple exposed areas and its relationship to teeth extraction. Study of 20 cases. Oral Oncolgy 2006;42:327-9.

3. Shannon J, Shannon J, Modelevsk S, Grippo AA. Bisphohonates and osteonecrosis of the jaw. J Am Geriatr Soc 2011;59:2350-5.

4. Wang J, Goodger NM, Pogrel MA. Osteonecrosis of the jaws associated with cancer chemotherapy. J Oral Maxillofac Surg 2003;61:1104-7.

5. Merigo E, Manfredi M, Meleti M, Guidotti R, Ripasarti A, Zanzucchi E, et al. Bone necrosis of the jaws associated with bisphosphonate treatment: a report of twenty-nine cases. Acta Biomed 2006; 77:109-17.

6. Migliorati CA. Bisphosphonates and oral cavity avascular necrosis of bone. J Clin Oncol 2003;21: 4253-4.

7. Vahtsevanos K, Kyrgidis A, Verrou E, et al. Longitudinal cohort study of risk factors in cancer patients of bisphosphonate- related osteonecrosis of the jaw. J Clin Oncol 2009;27:5356-62.

8. Ellemann K, Sjogren P, Banning AM, Jensen D. Trial of intravenous lidocaine on painful neuropathy in cancer patients. Clin J Pain 1989;5:291-4.
9. Heaney RP, Yates AJ, Santora AC. Biphosphonate effects and the bone remodeling transient. J Bone Miner Res 1997;12:1143-51.

10. Allen MR, Burr DB. The pathogenesis of bisphosphonate- related osteonecrosis of the jaw: So many hypotheses, so few data. J Oral Maxillofac Surg 2009;67:61-70.

11. Hernández-Vigueras S, Jané-Salas E, Pérez Tomás R, López-López J. Osteonecrosis de maxilares asociada al uso de bifosfonatos: Revisión de 491 casos. Av Odontoestomatol 2012;28(4):199-209.

12. Rogers MJ. From molds and macrophages to mavelonate: a decade of progress in understanding the molecular mode of action of bisphosphonates. Calcif Tissue Int 2004;75:451-61.

13. Vannuchi AM, Ficarra G, Antonioli E, Bosi A. Osteonecrosis of the jaw associated with zoledronate therapy in a patient with multiple myeloma. Br J Haematol 2005;128:738.

14. Alonci A, Allegra A, Bellomo G, Quartarone E, Oteri G, Nastro E, et al. Patients with bisphosphonateassociated osteonecrosis of the jaw have unmodified levels of soluble vascular endothelial growth factor receptor 1. Leuk Lymphoma 2007;48:1852-4.

15. Oteri G, Allegra A, Bellomo G, Alonci A, Nastro E, Penna G, et al. Reduced serum levels of Interleukin 17 in patients with osteonecrosis of the jaw and in multiple myeloma subjects after bisphosphonates administration. Cytokine 2008; 43:103-4.

16. Malden N, Beltes C, Lopes V. Dental extractions and bisphosphonates: the assessment, consent and management, a proposed algorithm. Br Dent J 2009;206:93-8.

17. Ruggiero SL, Dodson TB, Assael LA, Landesberg R, Marx RE, Mehrotra B. American association of oral and maxillofacial surgeons position paper on bisphosphonate-related osteonecrosis of the jaws2009 update. J Oral Maxillofac Surg 2009;67:2-12.

18. Advisory Task Force on Bisphosphonate-Related Ostenonecrosis of the Jaws. American Association 
of Oral and Maxillofacial Surgeons position paper on bisphosphonate-related osteonecrosis of the jaws. J Oral Maxillofac Surg 2007;65:369-76.

19. Khan AA, Morrison A, Hanley DA, Felsenberg D, McCauley LK, et al. Diagnosis and management of osteonecrosis of the jaw: a systematic review and international consensus.J Bone Miner Res 2015;30:3-23.

20. Sanchís JM, Bagán JV, Murillo J, Díaz JM, Asensio L. Risk of developing BRONJ among patients exposed to intravenous bisphosphonates following tooth extraction. Quintessence Int 2014;45:769-77.

21. Marx R, Cillo J, Ulloa J. Oral bisphosphonatesinduced osteonecrosis: Risk factors. Prediction of risk using serum CTX testing, prevention and treatment. J Oral Maxillofac Surg 2007;61:2397410.

22. Hutcheson A, Cheng A, Kunchar R, Stein B, Sambrook P, Goss A. A C-terminal crosslinking telopeptide test-based protocol for patients on oral bisphosphonates requiring extraction: a prospective single-center controlled study. J Oral Maxillofac Surg 2014 Aug;72:1456-62.
23. Ruggiero SL, Dodson TB, Fantasia J, Gooday R, Aghaloo T, et al. American Association of Oral and Maxillofacial Surgeons Position Paper on Medication-Related Osteonecrosis of the jaw: 2014 Update. J Oral Maxillofac Surg 2014;72: 1938-56.

24. Hinchy NV, Jayaprakash V, Rossitto RA, Anders PL, Korff KC, et al. Osteonecrosis of the jaw: Prevention and treatment strategies for oral health professionals. Oral Oncol 2013;49:878-86.

25. Montefusco V, Gay F, Spina F. Antibiotic profilaxis before dental procedures may reduce the incidence of osteonecrosis of the jaw in patients with multiple myeloma treated with bisphosphonates. Leukemia and Lymphoma 2008;49:2156-62.

\section{CORRESPONDENCIA}

Prof. José María Martínez-González

Departamento de Medicina y Cirugía Bucofacial Facultad de Odontología

Universidad Complutense de Madrid

Correo electrónico: jmargo@ucm.es 